\section{Revisiting the global detection capability of earthquakes during the period immediately after a large earthquake: considering the influence of intermediate-depth and deep earthquakes}

\section{Takaki Iwata}

Waseda University, Tokyo, Japan

\section{Abstract}

This study examines the global earthquake detection capability of the Global Centroid Moment Tensor (GCMT) catalogue during the periods immediately following large earthquakes, including intermediate-depth (70 $\leq$ depth $<300 \mathrm{~km}$ ) and deep (300 km $\leq$ depth) events. We have already shown that the detection capability beyond an aftershock zone degrades remarkably and that this condition persists for several hours after the occurrence of large shallow (depth $<70 \mathrm{~km}$ ) earthquakes. Because an intermediate-depth or deep earthquake occasionally generates seismic waves with significant amplitudes, it is necessary to investigate the change in the detection capability caused by such events. To this end, from the GCMT catalogue, we constructed the time sequences of the earthquakes that occurred immediately after the large earthquakes, and stacked these time sequences. To these stacked sequences, we then applied a statistical model representing the magnitude-frequency distribution of all observed earthquakes. This model has a parameter that characterizes the detection capability, and the temporal variation of the parameter is estimated by means of a Bayesian approach with a piecewise linear function. Consequently, we find that the global detection capability is lower after the occurrence of shallow earthquakes with magnitudes $\geq 5.45$, intermediate-depth earthquakes with magnitudes $\geq 5.95$, and deep earthquakes with magnitudes $\geq 6.95$.

\section{Introduction}

Evaluating the detection capability of an earthquake catalogue is the first step of statistical seismicity analysis, and this has stimulated several studies on regional ${ }^{1,2}$ and global $^{3}$ catalogues. In particular, it is important to closely examine the temporal homogeneity of a catalogue because, occasionally, we investigate temporal changes in the properties of seismic activity such as the $b$-value ${ }^{4}$ or seismicity rate. ${ }^{5}$ We may arrive at an incorrect conclusion in such studies if we fail to accurately evaluate the detection capability.

It is well known that a temporal change in detection capability accompanies the occurrence of a large earthquake. Often, events that occur in the period immediately after a large earthquake go undetected, and there are two reasons for this. First, when many aftershocks occur within a short time period, their seismic wave records overlap. Second, when a mainshock occurs, the corresponding large amplitudes in the wave data obscure subsequent earthquakes.

The second reason demands serious consideration with respect to the Global Centroid Moment Tensor (GCMT) catalogue, which is regarded to be the most reliable worldwide earthquake catalogue. A notable characteristic of the GCMT catalogue is that the focal parameters are determined by waveform inversion, ${ }^{6,7}$ and not by the phase arrival times. This characteristic contributes to its homogeneity in space and time, and therefore the GCMT catalogue is frequently used in research on global seismic activity.

However, this characteristic also result in a disadvantage. A large earthquake generates seismic waves, and they contaminate the wave records of subsequent earthquakes, thus disturbing the inversion procedure. Because the waves of a large earthquake can be detected on the opposite side of the globe from the epicenter, the capability of detecting not only aftershocks but also global earthquakes will degrade.

To quantify this degradation, Iwata ${ }^{8}$ (hereafter referred to as IW2008) carried out an analysis based on a Bayesian approach and showed that the global detection capability of the GCMT catalogue degrades significantly after a large shallow earthquake. In this scenario, the period of lower capability lasts from a few hours to half a day after the occurrence of a shallow earthquake with a magnitude equal to or greater than 5.45 .

IW2008 ${ }^{8}$ investigated the reduced detection capability only during periods immediately following large shallow earthquakes because, in general, seismic waves produced by a shallow event have larger amplitudes than those produced by a deep one. ${ }^{9}$ However, a great deep earthquake, such as the 1994 Bolivian earthquake, can excite waves with significant amplitudes and long duration; ${ }^{10}$ therefore, we need to examine the possibility that a deep event may also lower the detection capability of a catalogue. Thus, the present study aims to carry forward the work of IW $2008^{8}$ by also considering the effects of intermediate-depth $(70 \leq$ depth $<300$ $\mathrm{km})$ and deep (300 km $\leq$ depth) earthquakes on the detection capability of catalogues in the period immediately following the large earthquakes.
Correspondence: Takaki Iwata, Now at Institute of Statistical Mathematics, 10-3 Midori-cho, Tachikawa, Tokyo 190-8562, Japan.

Tel. +81.50.5533.8445 - Fax: +81.42.526.4339.

E-mail: iwata@ism.ac.jp

Key words: seismicity, statistical methods, magnitude frequency, earthquake catalogue completeness, obstacle earthquake, Bayesian analysis.

Conflict of interest: the author declares no potential conflict of interest.

Acknowledgments: the author thanks Hiroshi Tsuruoka who inspired this study and Mitsuhiro Matsu'ura for useful discussions. The author also thanks an anonymous reviewer whose comments improved this paper and the editor Gerassimos A. Papadopoulos for his assistance in evaluating it. This study was partially supported by the Grantsin-Aid 23240039 for Scientific Research (A), and 22700295 for Young Scientists (B), by the Ministry of Education, Culture, Sports, Science and Technology, Japan, by the ISM Cooperative Research Program (2011-ISM.CRP-2007), and by the Waseda University Grant for Special Research Projects (Project number: 2009B-322). For this study, the author used the supercomputer system of the Institute of Statistical Mathematics.

Received for publication: 22 November 2011. Revision received: 26 January 2012.

Accepted for publication: 27 January 2012.

This work is licensed under a Creative Commons Attribution NonCommercial 3.0 License (CC BYNC 3.0).

(C) Copyright T. Iwata, 2012

Licensee PAGEPress, Italy

Research in Geophysics 2012; 2:e4

doi:10.4081/rg.2012.e4

\section{Compiling earthquake sequences in the period immediately after large earthquakes}

The global earthquake data used in this study were retrieved from the GCMT catalogue; the data span the period from January 1977 to December 2010. This catalogue provides seismic moment to express the size of an earthquake, and we converted the value of the moment $M$ to the moment magnitude $M_{w}$ using the relationship given by Kanamori: ${ }^{11}$

$$
M_{w}=2 / 3 \cdot\left(\log _{10} M-9.1\right) .
$$

In this study, a large earthquake whose seismic waves obstruct the waveform inversion for subsequent earthquakes is referred to as an obstacle earthquake. Events whose $M_{w}$ and depth $d$ within specific ranges were selected as possible obstacle earthquakes, and we con- 
structed time sequences of the earthquakes that occurred in the period immediately following each of the obstacle earthquakes. As mentioned above, the main interest of this study is the capability of detecting global earthquakes outside an aftershock zone. Therefore, in the construction of the sequences, we disregarded earthquakes located close to the obstacle earthquakes. Kagan ${ }^{12}$ provided a formula that expresses the length $L$ of the focal zone of an earthquake in terms of $M_{w}$, as follows:

$$
\log _{10} L=-1.43+0.473 M_{w}
$$

This equation holds for global shallow $(d \leq$ $70 \mathrm{~km}$ ) earthquakes, and is similar to the formula given in another study. ${ }^{13}$ In this study, $5 \mathrm{~L}$ is considered to be the radius of the aftershock zone to ensure complete removal of aftershocks. In addition, the location uncertainty in the GCMT catalogue was taken into account. Engdahl et al. ${ }^{14}$ and Kagan ${ }^{15}$ reported that the average location uncertainty is roughly $40 \mathrm{~km}$, with a standard deviation of approximately 20 $\mathrm{km}$. Therefore, in each of the sequences, we excluded earthquakes occurring within $5 L+60$ $\mathrm{km}$ of the obstacle earthquake.

Next, by shifting each of the obstacle earthquakes to time zero, we stacked the constructed sequences. We considered four magnitude ranges of the obstacle earthquakes: $5.45 \leq M_{w}$ $<5.95,5.95 \leq M_{w}<6.45,6.45 \leq M_{w}<6.95$, and $6.95 \leq M_{w}$. In ordinary cases, the magnitude is rounded off to the first decimal place, and hence the four magnitude ranges are essentially the same as those of $5.5 \leq M_{w}<6.0,6.0$ $\leq M_{w}<6.5$, and so on. Three ranges of depths were also considered: $d<70 \mathrm{~km}$ (shallow), 70 $\leq d<300 \mathrm{~km}$ (intermediate-depth), and 300 $\mathrm{km} \leq d$ (deep). Therefore, all of the obstacle earthquakes were categorized into 12 groups, depending on the magnitude and depth, and stacked sequences were constructed for each of the groups.

Figure 1 shows the magnitude-time histories for earthquakes in the 12 sequences. In several cases, we find clear defects of relatively small earthquakes close to time zero (i.e. the occurrences of the obstacle earthquakes) providing explicit evidence of the decrease in detection capability. One point to note with regard to this figure is that the defects are observed after not only the shallow obstacle earthquakes but also the intermediate-depth and deep earthquakes, suggesting that the occurrence of an intermediate-depth or deep earthquake lowers the detection capability.

\section{Statistical evaluation of the capability of detecting earthquakes}

For a quantitative evaluation of the capability of detecting earthquakes, this study used a probability density function $f(M)$ of a magnitude-frequency distribution of all the observed earthquakes; the probability density function was introduced by Ogata and Katsura. ${ }^{16}$ The function $f(M)$ is represented by a product of two probability density functions. The first function is an exponential distribution: $w(M \mid \beta)=\beta \exp (-\beta M)$.

This distribution is equivalent to the Gutenberg-Richter (GR) law ${ }^{17}$ which is the most widely accepted model of the magnitudefrequency distribution of earthquakes, and the parameter $\beta$ is related to the $b$-value of the GR law by the relationship $\beta=b \ln 10$. The second is the detection rate of earthquakes $q(M)$, the percentage of earthquakes actually detected at magnitude $M$ by a seismic network. As a plausible function for the detection rate, the cumulative distribution function of a normal distribution

$q(M \mid \mu, \sigma)=\int_{-\infty}^{M} \frac{1}{\sqrt{2 \pi} \sigma} \exp \left[-\frac{(x-\mu)^{2}}{2 \sigma^{2}}\right] d x$

has been already suggested and used in several studies, $3,16,18$ and the present study follows these studies.

Normalizing $w(M) q(M)$ provides the target probability density function as follows:

$$
\begin{aligned}
f(M \mid \beta, \mu, \sigma) & =\frac{w(M \mid \beta) q(M \mid \mu, \sigma)}{\int_{-\infty}^{\infty} w(M \mid \beta) q(M \mid \mu, \sigma) d M} \\
& =\frac{\exp (-\beta M) q(M \mid \mu, \sigma)}{\int_{-\infty}^{\infty} \exp (-\beta M) q(M \mid \mu, \sigma) d M} .
\end{aligned}
$$

The likelihood function of the parameters, which measures the goodness-of-fit of the above model, is given by

$$
L(\beta, \sigma, \mu)=\prod_{i=1}^{N} f\left(M_{i} \mid \beta, \mu, \sigma\right),
$$

where $M_{i}$ denotes the magnitude of the $i$ th earthquake and $N$ is the number of analyzed earthquakes. Figure 2 shows that this statistical model fits well with the magnitude distribution of all the shallow earthquakes compiled in the GCMT catalogue (see also Figure 1 of IW2008 ${ }^{8}$ ). In this figure, the model curve was drawn using the estimated parameters obtained through the maximization of the loglikelihood function $L(\beta, \sigma, \mu)$ (i.e. the maximum likelihood method).

According to Equation 4, $\mu$ denotes the magnitude at which $50 \%$ of all earthquakes are expected to be detected; a higher value of $\mu$ implies lower detection capability. In the eval-

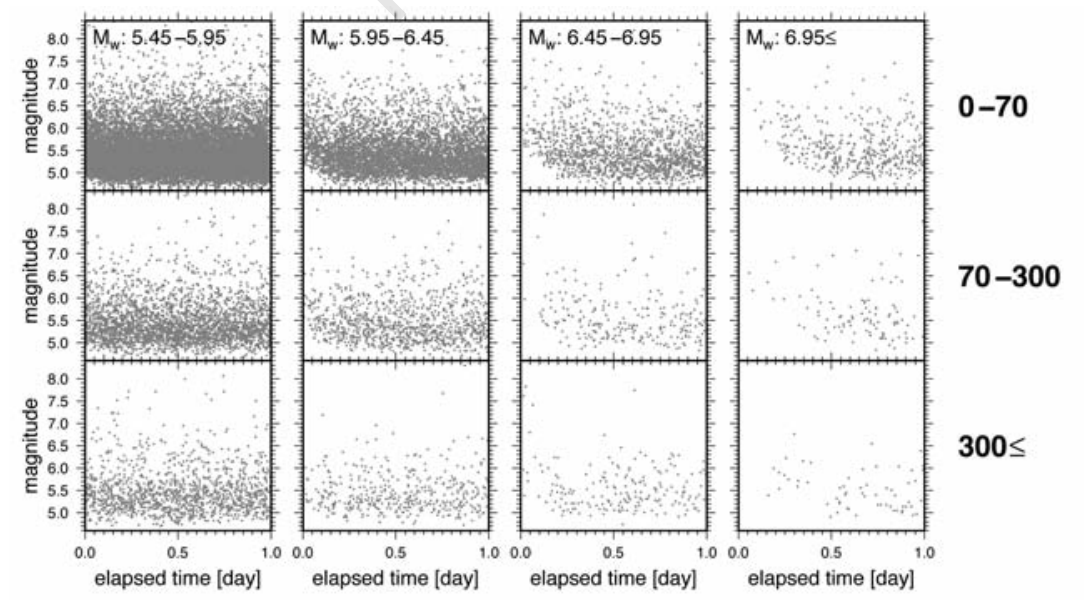

Figure 1. Magnitude-time histories for earthquakes occurring after the obstacle earthquakes.

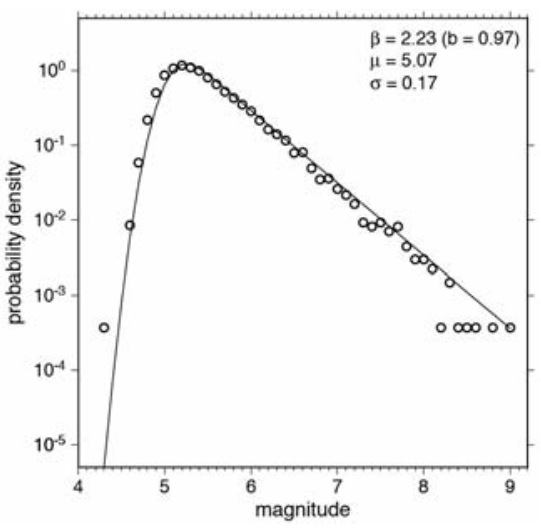

Figure 2. Probability density function of the magnitudes of all the shallow $(d<70$ $\mathrm{km}$ ) earthquakes compiled in the GCMT catalogue (open circles) and the model curve of the model (solid line), which is given by Equation 5, fitted with the data. Best estimates of the parameters are shown in the top right corner. 
uation of the detection capability, $\mu$ is the most fundamental among the three parameters and, therefore, the present study examines the temporal variation in $\mu$ after the occurrence of obstacle earthquakes.

The temporal variation in $\mu$ was estimated through a Bayesian method used in IW2008. The temporal variation is represented by a piecewise linear function or linear spline, ${ }^{19}$ and we optimized the variation with a smoothness constraint so that it does fit the data. The details are shown in the Appendix and IW2008. ${ }^{8}$

The significance of the temporal variation is measured by a comparison of the Akaike Bayesian Information Criterion ${ }^{20}$ (ABIC); the smaller the ABIC value, the better the model fits the data. Note that in the model where we do not allow $\mu$ to vary with time, the Bayesian approach essentially agrees with the maximum likelihood method and ABIC should be referred to as the Akaike Information Criterion ${ }^{21}$ (AIC) as per convention. However, for simplicity, both $\mathrm{ABIC}$ and AIC are uniformly written as ABIC in this manuscript (see Appendix for the equivalence of ABIC and AIC).

\section{Results and Discussion}

Figure 3 shows the estimated temporal variations in $\mu$ for the 12 datasets. Among these 12 cases, 8 show an increase in $\mu$ value immediately after the occurrence of the obstacle earthquakes. A comparison of ABIC values confirms this. Table 1 lists the ABIC values of the models without temporal variation in $\mu$ minus those of the models with variation; a positive value indicates that the model with variation fits the data better than the one without variation. As seen in this table, the 8 cases in which temporal variation in $\mu$ was found in Figure 3 show positive values.

To summarize Figure 3 and Table 1, the detection capability is seen to decrease after the occurrence of shallow earthquakes with $M_{w}$ $\geq 5.45$, intermediate-depth earthquakes with $M_{w} \geq 5.95$, and deep earthquakes with $M_{w} \geq$ 6.95 ; the lower limit of the magnitude range increases with the depth of the obstacle earthquakes. This is consistent with theoretical expectation: according to seismic wave theory, ${ }^{9}$ surface waves are dominant at great distances and the amplitudes of the waves generally decrease as the source depth is deeper.

From the estimated variation in $\mu, P\left(M_{\mathrm{th}}\right)$, we computed the probability of undetected earthquakes with magnitudes equal to or greater than $M_{\mathrm{th}}$ :

$$
P\left(M_{\mathrm{th}}\right)=\frac{\int_{M_{\mathrm{th}}}^{\infty} \exp (-\beta M)[1-q(M \mid \mu, \sigma)] d M}{\int_{M_{\mathrm{th}}}^{\infty} \exp (-\beta M) d M} .
$$

Figure 4 shows the temporal change in the probability for $M_{\mathrm{th}}=5.45,5.75$, and 5.95 .
Kagan $^{15}$ reported that the magnitude completeness threshold in the GCMT catalogue is between 5.2 and 5.8. Even when we make the most conservative choice from the above range of the completeness threshold, some earthquakes are not detected, with a probability of a few percent to a few tens of percent, over a period of several hours after a large $\left(M_{w} \geq 6.95\right)$, shallow, or intermediate-depth earthquake.

The probability of an undetected earthquake is significant immediately after the occurrence
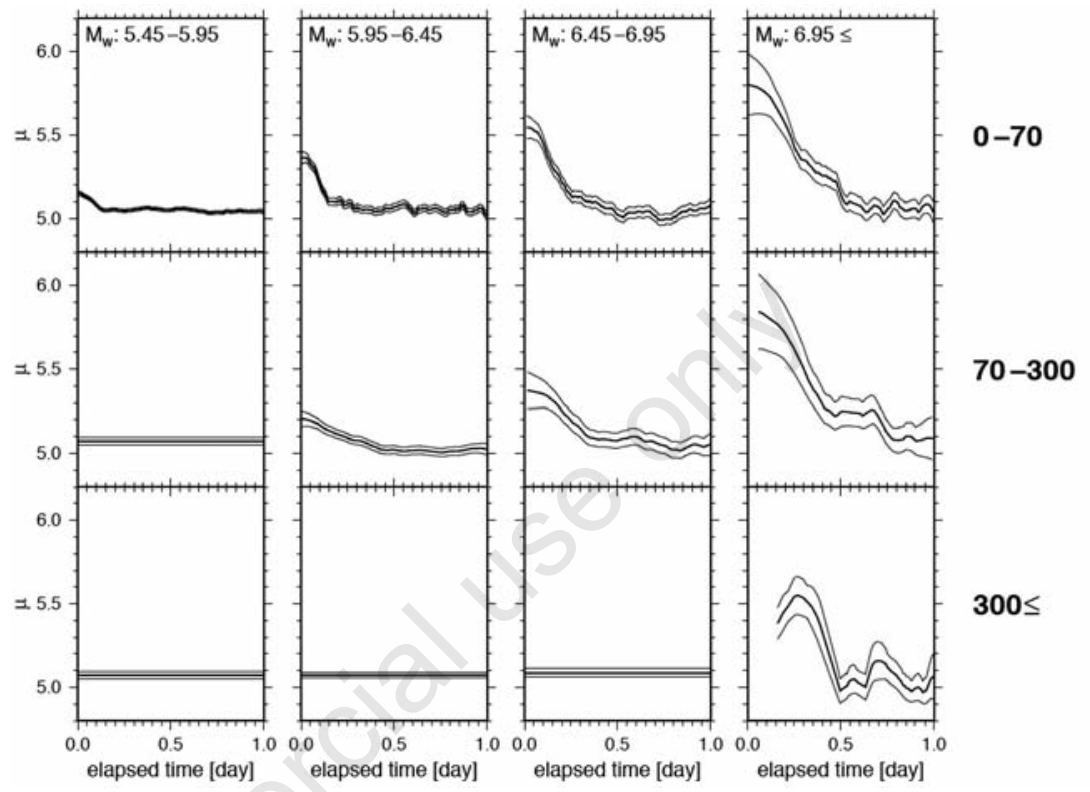

Figure 3. Estimated temporal variations in $\mu$ (bold lines) and their two standard error bands (thin lines). In some of the cases, the errors of the estimation are very small, and hence the bold and solid lines are indistinguishable.

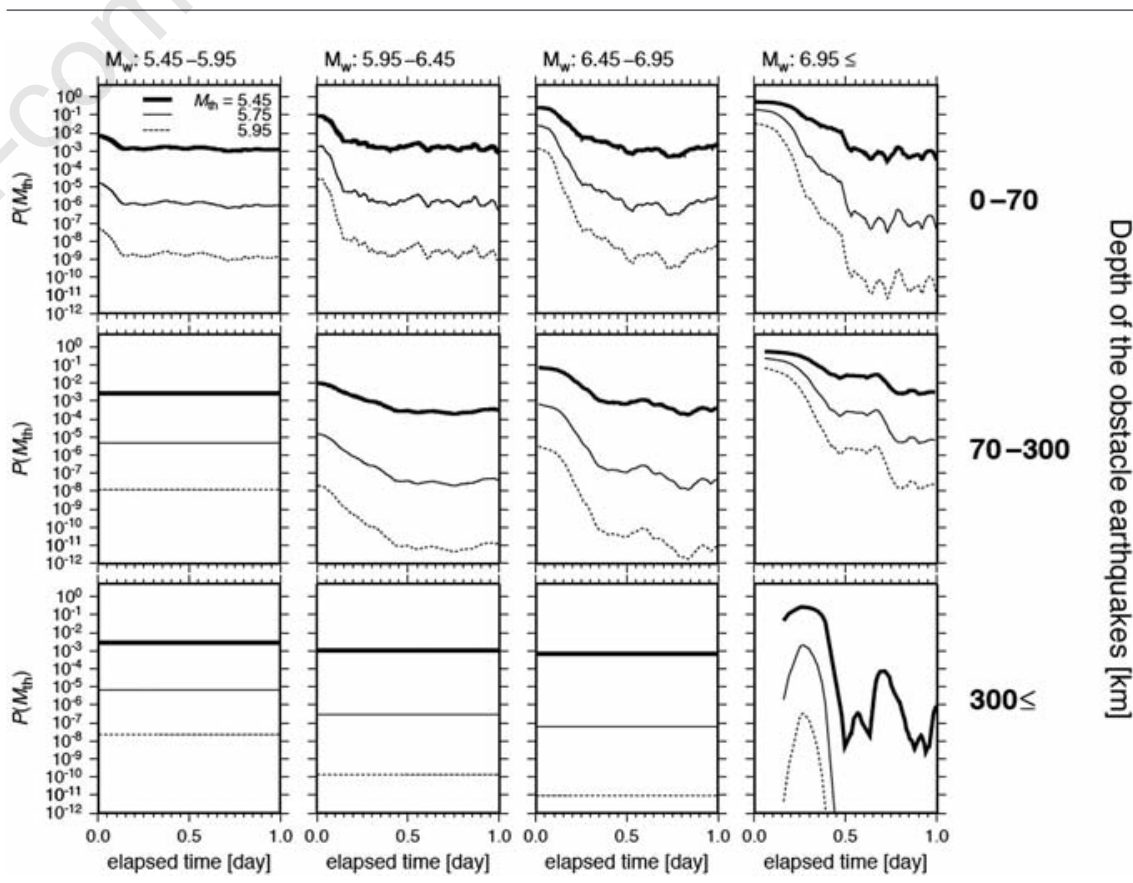

Figure 4. Computed probability of missing earthquakes of magnitude larger than $M_{\text {t }}$ $=5.45$ (bold line), 5.75 (thin line), and 5.95 (dotted line) as a function of the time elapsed since the occurrence of the obstacle earthquakes. 
Table 1. Model comparison using ABIC values. Positive values correspond to the cases in which the model with temporal variation in $\mu$ fits the data better than the one without variation.

\begin{tabular}{|c|c|c|c|c|}
\hline \multicolumn{5}{|c|}{ Magnitude } \\
\hline Depth (km) & $5.45-5.95$ & $5.95-6.45$ & $6.45-6.95$ & $6.95 \leq$ \\
\hline $0-70$ & 68.4 & 147.5 & 87.3 & 37.5 \\
\hline $70-300$ & -2.0 & 13.8 & 2.1 & 1.4 \\
\hline $300 \leq$ & -2.0 & -2.0 & -2.0 & 3.4 \\
\hline
\end{tabular}
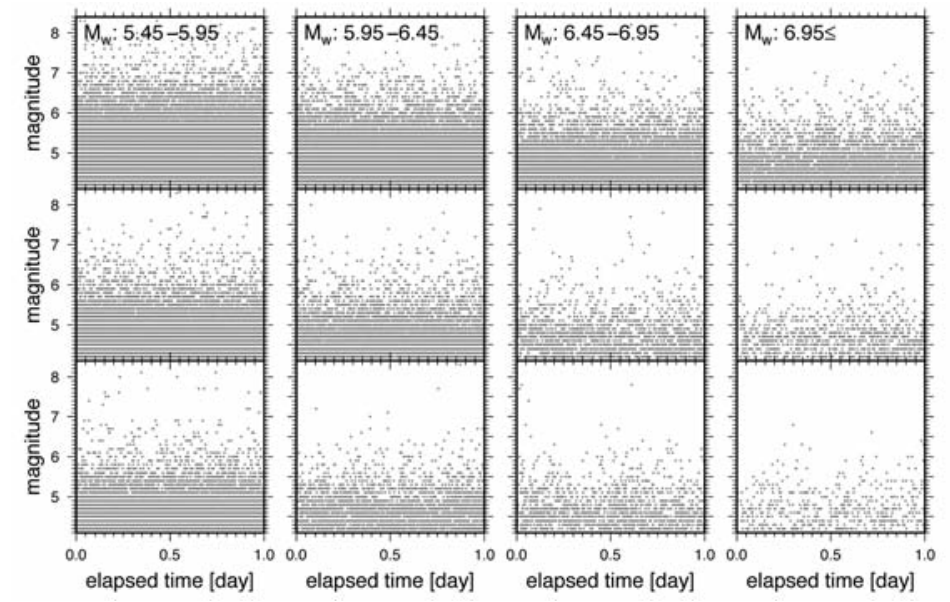

$0-70$

$300 \leq$

$70-300$

Figure 5. Magnitude-time histories for earthquakes recorded in the PDE catalogue occurring after the obstacle earthquakes.

ing the obstacle earthquakes retrieved from the GCMT catalogue. Figure 5 shows magnitude-time plots for the PDE catalogue drawn using the official magnitude of the catalogue (details available from: http://earthquake.usgs. gov/earthquakes/eqarchives/epic/codemagnitude.php). In contrast to Figure 1, we do not find any remarkable defects of small earthquakes around time zero. The earthquake parameters in the PDE catalogue are determined by phase picking, and not waveform inversion; thus, the contamination of seismic waves does not seriously affect the recorded data beyond the aftershock zone. The combined use of the PDE catalogue, or another catalogue based on phase arrival times and the GCMT catalogue would be beneficial in an analysis of short-term seismicity as it may prevent an unexpected bias in the result.

One might point out that, to compare the capabilities of the GCMT and PDE catalogues quantitatively, we should apply the statistical model described in this study also to the PDE catalogue. As seen in Figure 6, however, the magnitude-frequency distribution of all the shallow earthquakes listed in the PDE catalogue during the period from January 1977 to December 2010 has a complex feature, and the statistical model described by Equation 5 is inapplicable; another approach must be established to examine the detection capability of the PDE catalogue.

\section{References}

1. Nanjo KZ, Ishibe T, Tsuruoka $\mathrm{H}$, et al. Analysis of the completeness magnitude and seismic network coverage of Japan. Bull Seism Soc Am 2010;100:3261-68.

2. Schorlemmer D, Woessner J. Probability of detecting an earthquake. Bull Seism Soc Am 2008;98:2103-17.

3. Woessner J, Wiemer S. Assessing the quality of earthquake catalogues: Estimating the magnitude of completeness and its uncertainly. Bull Seism Soc Am 2005; 95:684-98.

4. Utsu T. Statistical features of seismicity. In: WHK Lee, et al. (eds.) International handbook of earthquake and engineering seismology, Int Geophys Ser vol. 81. Amsterdam: Academic Press; 2002. pp. 719-32.

5. Marsan D, Nalbant SS. Methods for measuring seismicity rate changes: A review and a study of how the Mw 7.3 Landers earthquake affected the aftershock sequence of the Mw 6.1 Joshua Tree earthquake. Pure Appl Geophys 2005;162:115185 .

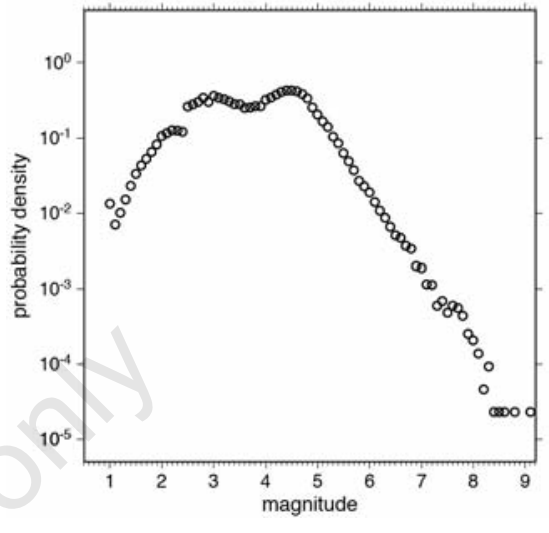

Figure 6. Probability density function of the magnitudes of all the shallow earthquakes listed in the PDE catalogue during the interval of January 1977 to December 2010.

6. Dziewonski AM, Chou T-A, Woodhouse JH. Determination of earthquake source parameters from waveform data for studies of global and regional seismicity. J Geophys Res 1981:86:2825-52.

7. Dziewonski AM, Woodhouse JH. An experiment in systematic study of global seismicity: Centroid-moment tensor solutions for 201 moderate and large earthquakes of 1981. J Geophy Res 1983;88:3247-71.

8. Iwata T. Low detection capability of global earthquake after the occurrence of large earthquakes: Investigation of the Harvard CMT catalog. Geophys J Int 2008;174:84956.

9. Aki K, Richards PG. Quantitative seismology. 2nd ed. San Francisco: University Science Books; 2002.

10. Kanamori H. Shaking without quaking. Science 1998;279:2063-4.

11. Kanamori H. The energy release in great earthquakes. J Geophy Res 1977;82:29817.

12. Kagan YY. Aftershock zone scaling. Bull Seism Soc Am 2002;92:641-55.

13. Henry C, Das S. Aftershock zones of large shallow earthquakes: fault dimensions, aftershock area expansion and scaling relations. Geophys J Int 2001;147:272-93.

14. Engdahl R, van der Hilst R, Buland R. Global teleseismic earthquake relocation 
with improved travel times and procedures for depth determination. Bull Seism Soc Am 1998;88:722-43.

15. Kagan YY. Accuracy of modern global earthquake catalogs. Phys Earth Planet Inter 2003;135:173-209.

16. Ogata Y, Katsura K. Analysis of temporal and spatial heterogeneity of magnitude frequency distribution inferred from earthquake catalogues. Geophys J Int 1993;113:727-38.

17. Gutenberg B, Richter RC. Frequency of earthquakes in California. Bull Seism Soc Am 1944;34:185-8.

18. Ringdal F. On the estimation of seismic detection thresholds. Bull Seism Soc Am 1975;65:1631-42.

19. Powell MJD. Approximation theory and methods. Cambridge University Press, New York, 1981.

20. Akaike H. Likelihood and Bayes procedure. In: JE Bernardo, et al. (ed.) Bayesian Statistics. Valencia: University Press; 1980. pp. 143-66.

21. Akaike H. A new look at the statistical model identification. IEEE Trans Autom Control 1974;19:716-23.

22. Good IJ, Gaskins RA. Nonparametric roughness penalties for probability densities. Biometrika 1971;58:255-77.
23. Eggermon PPB, La Riccia VN. Maximum penalized likelihood estimation, vol I. Density estimation. New York: Springer; 2001.

24. Good IJ. The estimation of probabilities. Cambridge: The MIT Press; 1965.

25. Kass RE, Raftery AE. Bayes factors. J Am Stat Assoc 1995;90:773-95.

26. Tierney L, Kadane JB. Accurate approximations for posterior moments and marginal densities. J Am Stat Assoc 1986; 81:82-6.

27. Akaike H. Factor analysis and AIC. Psychometrika 1987;52:317-32. 